\title{
Remarks on the Relation between the Lee-Yang Circle Theorem and the Correlation Inequalities
}

\author{
Keiichi R. Ito \\ Research Institute for Mathematical Sciences and Department of Physics, \\ Kyoto University, Kyoto, Japan
}

\begin{abstract}
We investigate the relation between the Lee-Yang circle theorem and the correlation inequalities. These results are general and independent of models. General properties of the partition functions which belong to the Lee-Yang class are given.
\end{abstract}

\section{Introduction}

Recently several authors have investigated the Euclidean boson quantum field models [the so-called $P(\phi)_{d}$-models] as a classical statistical mechanics $[1,2]$. In these articles we see that the Lee-Yang circle theorem and the correlation inequalities play a central role in the study. On the other hand, Griffiths et al. conjectured that a set of correlation inequalities determine the forms of the interactions $[3,4]$. From the points of view of these applications and conjectures, it is an interesting problem to decide the partition functions which satisfy the Lee-Yang circle theorem or the desired correlation inequalities.

Moreover, Newman recently proved that the Lee-Yang circle theorem leads to some correlation inequalities [5]. Therefore it is also an interesting problem to discuss the relation between the Lee-Yang circle theorem and the correlation inequalities. Finally we investigate the general properties of partition functions which satisfy the Lee-Yang circle theorem, as they have not appeared elsewhere.

We organize the paper as follows: In Section 2, we define classes of the partition functions $\mathscr{P}_{e}, \mathscr{L}, \mathscr{D}, \mathscr{I}$, and summarize the relevant correlation inequalities without proof. In Section 3, we investigate the Griffiths first (G-1) and the second (G-2) inequalities and discuss the relation between these inequalities and the Lee-Yang circle theorem. In Sections 4 and 5, we investigate the Griffiths-Hurst-Sherman inequality (GHS inequality) and the Lebowitz inequality. In Section 6, general properties of the partition functions which belong to the Lee-Yang class are given. 


\section{Classes $\mathscr{P}, \mathscr{L}, \mathscr{J}, \mathscr{D}$}

We summarize notations and definitions used below following [6]:

$D$ : unit disk $=\{z \in C ;|z| \leqq 1\}$

$\partial D$ : boundary of $D=\{z \in C ;|z|=1\}$

$D^{0}$ : interior of $D=D \backslash \partial D=\{z \in C ;|z|<1\}$

$D^{c}$ : exterior of $D=C \backslash D=\{z \in C ;|z|>1\}$

$\mathscr{P}_{e}^{(n)}$ or $\mathscr{P}_{e}$ : polynomials of $n$-variables $z_{1}, \ldots, z_{n}$ which are linear with respect to each $z_{i}$, and satisfy the evenness condition $P\left(z_{1}^{-1}, \ldots, z_{n}^{-1}\right)=P\left(z_{1}, \ldots, z_{n}\right) \prod z_{i}^{-1}$ with $P(0, \ldots, 0)=1$.

For the sake of the brevity, we restrict ourselves to the case in which all the coefficients are real. Thus $P \in \mathscr{P}_{e}$ is typically given by

$$
\begin{aligned}
P\left(z_{1}, \ldots, z_{n}\right)= & \left(1+z_{1}, \ldots, z_{n}\right)+\sum \beta_{i}^{(1)}\left(z_{i}+z_{1} z_{2} \ldots \hat{z}_{i} \ldots z_{n}\right) \\
& +\sum \beta_{i j}^{(2)}\left(z_{i} z_{j}+z_{1} \ldots \hat{z}_{i} \ldots \hat{z}_{j} \ldots z_{n}\right)+\ldots
\end{aligned}
$$

with

$$
\beta_{i_{1} i_{2} \ldots i_{l}}^{(l)} \in R .
$$

Here $\hat{z}_{i}$ (or $\hat{i}$ ) means that the variable $z_{i}$ is omitted. $\mathscr{L}^{(n)}$ or $\mathscr{L}$; The Lee-Yang class included in $\mathscr{P}_{e}$. We say that a polynomial $P \in \mathscr{P}_{e}$ belongs to $\mathscr{L}$ provided that any root $z_{i}\left(z_{j} ; j \neq i\right)$ of $P\left(z_{1}, \ldots, z_{n}\right)=0$ satisfies $z_{i}\left(z_{j} ; j \neq i\right) \in D^{c}$ as a function of $z_{j}(j \neq i)$ when $z_{j} \in D(j \neq i)$ and $z_{k} \in D^{0}$ for some $k$.

Since $P \in \mathscr{P}_{e}$, we see $z_{i}\left(z_{j} ; j \neq i\right) \in D^{0}$ when $z_{j} \in \bar{D}^{c}(j \neq i)$ and $z_{k} \in D^{c}$. Obviously all the roots of $P(z, \ldots, z)=0$ lie on $\partial D$ if $P \in \mathscr{L}$. This is the famous Lee-Yang circle theorem.

$\mathscr{I}$ : Set of $P \in \mathscr{P}_{e}$ such that all the roots of $P(z, \ldots, z)=0$ lie on the unit circle $\partial D$.

These definitions are general and independent of models. In order to define class $\mathscr{D}$, we use the Ising model of spin $1 / 2$ where there are only ferromagnetic pair interactions:

$$
H_{\Lambda}=-\sum_{i<j} J_{i j}\left(s_{i} s_{j}-1\right) / 2-\sum h_{i}\left(s_{i}+1\right) / 2
$$

where $0 \leqq J_{i j} \leqq \infty$ and $s_{i}(i \in \Lambda)$ is a random variable at the lattice site $i \in \Lambda$ which takes the values \pm 1 . Let $P$ be the relevant partition function;

$$
P=\sum_{\left\{s_{i}= \pm 1\right\}} \exp \left(-H_{A}\right)
$$

with

$$
z_{i}=\exp \left(h_{i}\right) .
$$

Therefore $P$ is given by the coefficients

$$
\beta_{i_{1} i_{2} \ldots i_{l}}^{(l)}=\prod_{i=i_{1}}^{i_{l}} \prod_{j \in \Lambda ; j \notin\left\{i_{1} i_{2} \ldots i_{l}\right\}} \gamma_{i j}
$$

with

$$
\gamma_{i j}=\exp \left(-J_{i j}\right) .
$$

Then obviously $0 \leqq \gamma_{i j} \leqq 1$, however we extend this as $-1 \leqq \gamma_{i j} \leqq 1$, and denote the resultant set by $\mathscr{D}$. 
For $P \in \mathscr{P}_{e}$, we identify $P$ with its coefficients $\left\{\beta_{i_{1} i_{2} \ldots i_{l}}^{(l)}\right\} \in R^{d}\left(d=2^{n-1}-1\right)$, and consider the sets of functions $\mathscr{P}_{e}$ and $\mathscr{L}$ as the sets of the coefficients. In this sense, we denote the convex hulls of $\mathscr{L}, \mathscr{D}$ by $\hat{\mathscr{L}}$ and $\hat{\mathscr{D}}$ respectively, and the closure of $\mathscr{L}$ by $\overline{\mathscr{L}}$.

Finally in order to study the correlation inequalities, we sometimes restrict ourselves to the subsets where all the coefficients are real non-negative. We denote these by $\mathscr{P}_{e}^{+}, \mathscr{L}^{+}$, and $\mathscr{D}^{+}$respectively.

Now we define the so-called Ursell functions: for $P \in \mathscr{P}_{e}^{+}$, we define

$$
\left.\begin{array}{rl}
u^{(l)}\left(i_{1}, \ldots, i_{l}\right) & =\left(\prod_{i=i_{1}}^{i_{1}} z_{i} \partial / \partial z_{i}\right) \log P \quad l \geqq 2 \\
u^{(1)}(i) & =z_{i} \partial / \partial z_{i} \log P-1 / 2 .
\end{array}\right\}
$$

As is well known $\mathscr{D} \subset \overline{\mathscr{L}}\left(\mathscr{D}^{+} \subset \overline{\mathscr{L}}^{+}\right)$, and for $P \in \mathscr{D}^{+}$we see $[3,4,7-9]$ :

Griffiths first inequality; $u^{(1)}(i) \geqq 0$ for $z_{j} \geqq 1(j \in \Lambda)$

Griffiths second inequality; $u^{(2)}(i, j) \geqq 0$ for $z_{j} \geqq 1(j \in \Lambda)$

GHS inequality; $u^{(3)}\left(i_{1}, i_{2}, i_{3}\right) \leqq 0$ for $z_{j} \geqq 1(j \in \Lambda)$

Lebowitz inequality; $u^{(4)}\left(i_{1}, i_{2}, i_{3}, i_{4}\right) \leqq 0$ for $z_{j}=1(j \in \Lambda)$

Sylvester inequality; $u^{(6)}\left(i_{1}, \ldots, i_{6}\right) \geqq 0$ for $z_{j}=1(j \in \Lambda)$

where $\Lambda=\{1,2, \ldots, n\}$.

The following inequalities are conjectured by Newman for $P \in \mathscr{D}^{+}[5,8]$ :

$$
(-1)^{l-1} u^{(2 l)}\left(i_{1}, \ldots, i_{2 l}\right) \geqq 0 \quad \text { for } \quad z_{j}=1(j \in \Lambda)
$$

with $l=1,2, \ldots$.

Then we define the following classes of partition functions:

$C_{i}$ : The set of the partition functions $P \in \mathscr{P}_{e}^{+}$which satisfy the expected inequality for the $i$ th Ursell function.

\section{3. $\mathscr{L}^{+}$and $\mathbf{u}^{(1)}, \mathbf{u}^{(2)}$}

Lemma 1. Let $P \in \mathscr{L}^{+}$, then $u^{(1)}(i) \geqq 0$ provided $z_{j} \geqq 1(j \in \Lambda)$.

Proof. Let $P$ be given by

$$
P=B\left(z_{1}, \ldots, z_{n-1}\right)+A\left(z_{1}, \ldots, z_{n-1}\right) z_{n}
$$

where $A, B$ are linear functions of $z_{1}, \ldots, z_{n-1}$ with positive coefficients. $P \in \mathscr{L}$ implies

$$
|B / A| \leqq 1 \quad \text { provided } \quad\left|z_{i}\right| \geqq 1 \quad i=1,2, \ldots, n-1 .
$$

On the other hand,

$$
u^{(1)}(n)=\left(A z_{n}-B\right) / 2 P \text {. }
$$

Lemma 2. For $P \in \mathscr{P}_{e}$, (i) and (ii) hold:

(i) If $u^{(1)}(i) \geqq 0$ for $z_{k} \geqq 1(k \in \Lambda)$, then $u^{(2)}(i, j)_{\mid z=1} \geqq 0$.

(ii) If $u^{(2)}(i, j) \geqq 0$ for $z_{k} \geqq 1(k \in \Lambda)$, then $u^{(1)}(i) \geqq 0$ for $z_{k} \geqq 1(k \in \Lambda)$.

Proof. (i) Let all $z_{k}$ except $z_{j}$ be equal to 1. Since $P \in \mathscr{P}_{e}, u^{(1)}(i)=\left(z_{j}-1\right) f\left(z_{j}\right)$ where the $\mathrm{G}-1$ inequality ensures $f\left(z_{j}\right) \geqq 0$ for $z_{j} \geqq 1$. Thus $u^{(2)}(i, j)_{\mid z=1}=f(1) \geqq 0$.

(ii) Since $P \in \mathscr{P}_{e}, u^{(1)}(i)_{\mid z=1}=0$. 
However, unfortunately $P \in \mathscr{L}^{+}$does not necessarily imply the second Griffiths inequality with positive external fields, i.e., $P \in \mathscr{L}^{+}$does not imply

$$
u^{(2)}\left(i_{1}, i_{2}\right) \geqq 0 \quad \text { with } \quad z_{j} \geqq 1(j \in \Lambda) .
$$

An explicit counterexample is given in the next section.

Finally for $P \in \mathscr{L}^{+}$, we can show the correlation inequalities which correspond to $\left\langle s_{1} s_{2} \ldots s_{l}\right\rangle \geqq 0$ provided $h_{i} \geqq 0(i \in \Lambda)$. This is the $\mathrm{G}-1$ inequality in usual sense.

Theorem 1. Let $P\left(z_{1}, \ldots, z_{n}\right) \in \mathscr{L}^{+}$, then

$$
\prod_{i \in S}\left(z_{i} \partial / \partial z_{i}\right)\left[P\left(z_{1}, \ldots, z_{n}\right)\left(\prod_{i=1}^{n} z_{i}\right)^{-1 / 2}\right] \geqq 0
$$

provided $z_{i} \geqq 1(i \in \Lambda)$ where $S \subset \Lambda$ denotes the set of indices.

Proof. It is sufficient to consider the case that all the indices are different. Let $P$ be given by

$$
P=\sum_{\left\{i_{1}, i_{2}, \ldots, i_{l}\right\} \subset S} a_{i_{1}, i_{2} \ldots i_{l}} z_{i_{1}} z_{i_{2}} \ldots z_{i_{l}}
$$

where $\left\{a_{i_{1}, i_{2}}, \ldots, i_{l}\right\}$ are linear functions of $z_{j} \in \Lambda \backslash S$ with positive coefficients. Then

$$
\prod_{i \in S}\left(z_{i} \partial / \partial z_{i}\right)\left[P \prod_{i=1}^{n} z_{i}^{-1 / 2}\right]=\left[2^{|S|} \prod_{i=1}^{n} z_{i}^{1 / 2}\right]^{-1} Q,
$$

where

$$
\begin{aligned}
Q & =2^{|S|} Z^{S} P_{S}-2^{|S|-1} \sum_{i \in S} z^{S \backslash i} P_{S \backslash i}+2^{|S|-2} \sum_{i, j \in S} z^{S \backslash(i j)} P_{S \backslash(i, j)}+\ldots+(-1)^{|S|} P \\
& =\sum_{\left\{i_{1}, i_{2}, \ldots, i_{l}\right\}=I \subset S}(-1)^{|S|-|I|} a_{I^{\prime}} z^{I}
\end{aligned}
$$

with

$$
a_{I}=a_{i_{1}, i_{2}, \ldots, i_{l}} ; \quad z^{I}=\prod_{i \in I} z_{i} ; \quad P_{I}=\prod_{i \in I} \partial / \partial_{z_{i}} P .
$$

Let $z_{j} \in \Lambda \backslash S$ be fixed and $\geqq 1$, thus we study the necessary and sufficient condition that ensures $Q \geqq 0$ provided that $z_{i} \geqq 1(i \in S)$. Following Lemma 4, which is proved below, this is

$$
\begin{aligned}
& \text { (1) } a_{1,2, \ldots, l} \geqq 0 \\
& \text { (2) } a_{1,2, \ldots, l}-a_{1,2, \ldots, \hat{i}, \ldots, l \geqq 0}(i \in S), \\
& \text { (3) } a_{1,2, \ldots, l}-a_{1, \ldots, \hat{i}, \ldots, l}-a_{1, \ldots, \hat{j}, \ldots, l}+a_{1, \ldots, \hat{i}, \ldots, \hat{j}, \ldots, l} \geqq 0(i, j \in S), \\
& \cdot \cdot \cdot \cdot \cdot \cdot \cdot \cdot \cdot \cdot \cdot \\
& (l+1) a_{1,2, \ldots, l}-\sum_{i \in S} a_{1,2, \ldots, \hat{i}, \ldots, l}+\sum_{i, j \in S} a_{1, \ldots, \hat{i}, \ldots, \hat{j}, \ldots, l} \\
& +\ldots+(-1)^{l} a_{\hat{1}, \hat{2}, \ldots, \hat{l}} \geqq 0 .
\end{aligned}
$$

Here without loss of generality, we put $S=\{1,2,3, \ldots, l\} \subset \Lambda$.

These conditions are equivalent to

$$
(-1)^{|S \backslash I|} P_{I}\left(z_{j}=-1 ; j \in S \backslash I\right)=(-I)^{|S \backslash I|}\left(\prod_{i \in I} \partial / \partial z_{i}\right) P_{\mid z_{j}=-1 ; j \in S \backslash I} \geqq 0
$$

for any subset $I \subset S$. Since $P \in \mathscr{L}^{+}$, all the roots of $P_{I}\left(z_{j}=z ; j \in S \backslash I\right)=0$ lie in the unit disk $D$. Now we investigate the sign of $P_{I}(-1)$.

$$
\begin{aligned}
P_{I}(z) & =a_{S} z^{|S \backslash I|}+\ldots+a_{I}=\sum_{J ; I \subset J \subset S} a_{J} z^{|J \backslash I|} \\
& =a_{S} \prod_{i=1}^{r}\left[\left(z-\omega_{i}\right)\left(z-\bar{\omega}_{i}\right)\right] \prod_{j=1}^{|S \backslash I|-2 r}\left(z-\zeta_{j}\right)
\end{aligned}
$$


where $\left\{\omega_{i}, \bar{\omega}_{i}|\omega| \leqq 1\right\}$ are the complex roots, and $\left\{\zeta_{j} ;|\zeta| \leqq 1\right\}$ are the real roots of $P_{I}=0$. Since $a_{S}>0,\left(-1-\omega_{i}\right)\left(-1-\bar{\omega}_{i}\right)>0$ and $\operatorname{sgn} \prod_{j=1}^{|S \backslash I|-2 r}\left(-1-\zeta_{j}\right)=$ $\operatorname{sgn}(-1)^{|S \backslash I|}$, we see

$$
(-1)^{|S \backslash I|} P_{I}(-1) \geqq 0 \text {. }
$$

This completes the proof.

Finally we would like to point out that if $P \in \mathscr{L}, u^{(1)}(i)$ or $\left\langle s_{1} s_{2} \ldots s_{l}\right\rangle \propto \prod_{i \in S} z_{i} \partial / \partial z_{i}\left[P \prod z_{j}^{-1 / 2}\right]$ also satisfy the definition of $\mathscr{L}$ except the evenness condition $P\left(z_{1}^{-1}, \ldots, z_{n}^{-1}\right)=P\left(z_{1}, \ldots, z_{n}\right) \prod z_{i}^{-1}$. This is obvious because if $P\left(z_{1}, \ldots, z_{n}\right) \in \mathscr{L}, P\left(\exp \left(i \theta_{1}\right) z_{1}, \ldots, \exp \left(i \theta_{n}\right) z_{n}\right)$ with $\theta_{i} \in R$ again satisfies the definition of $\mathscr{L}$ except the evenness condition, and these correlation functions are essentially given by (3.2). However, this is not true for the higher order Ursell functions. In fact if it were true, the higher order Ursell functions would have definite signs in $\left\{z_{i} \geqq 1 ; i \in \Lambda\right\}$.

\section{4. $\mathscr{L}^{+}$and $\mathbf{u}^{(3)}, \mathbf{u}^{(4)}$}

In the cases of $n=1,2, \overline{\mathscr{L}}=\mathscr{D}\left(\overline{\mathscr{L}}^{+}=\mathscr{D}^{+}\right)$, and $C_{i}=\mathscr{D}^{+}=\mathscr{L}^{+},(i=1,2,3,4)$. In the case of $n=3$, we will easily see that $\overline{\mathscr{L}}=\hat{\mathscr{D}}\left(\overline{\mathscr{L}}^{+}=\hat{\mathscr{D}}^{+}\right)$, and $P \in \mathscr{L}^{+}$does not imply the desired inequality.

Lemma 3. Let $P \in \mathscr{P}_{e}$ be given

$$
P=1+z_{1} z_{2} z_{3}+\sum_{i=1}^{3} \beta_{i}\left(z_{i}+\partial / \partial z_{i}\left(z_{1} z_{2} z_{3}\right)\right) \text {. }
$$

Then $P \in \mathscr{L}$ if and only if

$$
\left|1 \pm \beta_{i}\right|>\left|\beta_{j} \pm \beta_{k}\right| \text {. }
$$

Proof. It is necessary and sufficient that

$$
\left(z_{3}\right)^{-1}=-\left[\beta_{3}+\beta_{2} z_{1}+\beta_{1} z_{2}+z_{1} z_{2}\right] /\left[1+\beta_{1} z_{1}+\beta_{2} z_{2}+\beta_{3} z_{1} z_{2}\right] \in D^{0}
$$

provided $z_{1}, z_{2} \in D$ and some of them $\in D^{0}$. Remark that the Shilov boundary of the polydisk $D \otimes D \otimes \ldots \otimes D$ is $\partial D \otimes \ldots \otimes \partial D$. Since $z_{3} \in \partial D$ provided $\left(z_{1}, z_{2}\right) \in \partial D \otimes \partial D$, the problem reduces to obtain a condition which is equivalent to

$$
P\left(z_{1}, z_{2}, z_{3}=0\right)=1+\beta_{1} z_{1}+\beta_{2} z_{2}+\beta_{3} z_{1} z_{2} \neq 0
$$

provided $\left(z_{1}, z_{2}\right) \in D \otimes D$.

Therefore,

$$
\text { Case (i) } \begin{array}{ll} 
& \beta_{2}=\beta_{3}=0: \\
& \left|\beta_{1}\right|<1 .
\end{array}
$$

Case (ii) $\beta_{2} \neq 0$ or $\beta_{3} \neq 0$ :

$\left|z_{2}\right|^{-1}=\left|\left(\beta_{2}+\beta_{3} z_{1}\right) /\left(1+\beta_{1} z_{1}\right)\right|<1 \quad$ provided $z_{1} \in D$.

This completes the proof. 
Now we investigate the correlation inequalities for $P \in \mathscr{L}^{+}$given in the previous Lemma 3. When all the arguments are different with each other, we have

$$
u^{(3)}(1,2,3)=z_{1} z_{2} z_{3} \prod \partial / \partial z_{i} \log P=z_{1} z_{2} z_{3} f_{3} / P^{3}
$$

where

$$
\begin{aligned}
f_{3} & =P^{2} P_{1,2,3}-P \sum P_{i} P_{j, k}+2 P_{1} P_{2} P_{3} \\
& =s_{0}\left(1-z_{1} z_{2} z_{3}\right)+s_{1}\left(z_{1}-z_{2} z_{3}\right)+s_{2}\left(z_{2}-z_{3} z_{1}\right)+s_{3}\left(z_{3}-z_{1} z_{2}\right)
\end{aligned}
$$

with

$$
\begin{aligned}
& s_{0}=1-\beta_{1}^{2}-\beta_{2}^{2}-\beta_{3}^{2}+2 \beta_{1} \beta_{2} \beta_{3}, \\
& s_{1}=\beta_{1}\left(1-\beta_{1}^{2}+\beta_{2}^{2}+\beta_{3}^{2}\right)-2 \beta_{2} \beta_{3}, \\
& s_{2}=\beta_{2}\left(1+\beta_{1}^{2}-\beta_{2}^{2}+\beta_{3}^{2}\right)-2 \beta_{3} \beta_{1}, \\
& s_{3}=\beta_{3}\left(1+\beta_{1}^{2}+\beta_{2}^{2}-\beta_{3}^{2}\right)-2 \beta_{1} \beta_{2} .
\end{aligned}
$$

\section{Lemma 4. Let}

$$
f=a_{0} z_{1} z_{2} \ldots z_{n}+\sum a_{i} z_{1} z_{2} \ldots \hat{z}_{i} \ldots z_{n}+\sum a_{i j} z_{1} z_{2} \ldots \hat{z}_{i} \ldots \hat{z}_{j} \ldots z_{n}+\ldots+a_{1,2, \ldots, n} .
$$

Then the necessary and sufficient condition so that $f \geqq 0$ provided $z_{i} \geqq 1(i \in \Lambda)$ is

$$
\begin{aligned}
& a_{0} \geqq 0 \\
& a_{0}+a_{i} \geqq 0(i \in \Lambda), \\
& a_{0}+a_{i}+a_{j}+a_{i, j} \geqq 0(i, j \in \Lambda), \\
& \cdot \cdot \dot{\cdot} \cdot \dot{\cdot} \cdot \dot{*} \cdot \cdot \cdot \cdot \\
& a_{0}+\sum a_{i}+\sum a_{i, j}+\ldots+a_{1,2, \ldots, n} \geqq 0 .
\end{aligned}
$$

Proof. Remark that $f$ is a linear function with respect to each variable. Therefore the necessary and sufficient condition so that $f \geqq 0$ for $z_{i} \geqq 1(i \in \Lambda)$ is

$$
\begin{aligned}
& f\left(z_{1}, z_{2}, \ldots, z_{n-1}, 1\right) \geqq 0, \\
& \partial / \partial z_{n} f\left(z_{1}, \ldots, z_{n}\right)=\partial / \partial z_{n} f\left(z_{1}, \ldots, z_{n}\right)_{\mid z_{n}=1} \geqq 0
\end{aligned}
$$

provided $z_{i} \geqq 1(i \in \Lambda)$. This discussion leads to the following condition:

for any $I \subset \Lambda, \quad\left(\prod_{i \in I} \partial / \partial z_{i}\right) f_{\mid z=1} \geqq 0$.

Theorem 2. For $P \in \mathscr{L}^{+}$

(i) $u^{(3)}(1,2,3) \leqq 0$ provided $z_{1}, z_{2}, z_{3} \geqq 1$ and $z_{4}=z_{5}=\ldots=z_{n}=1$.

(ii) $u^{(2)}(1,2)$ is not necessarily positive for $z_{i} \geqq 1(i \in \Lambda)$.

However, $u^{(2)}(1,2) \geqq 0$ provided $z_{1}, z_{2} \geqq 1$ and $z_{3}=z_{4}=\ldots=z_{n}=1$.

(iii) For $P \in \mathscr{P}_{e}^{+}$, let $\tilde{P}=P_{\mid z_{4}=\ldots=z_{n}=1}$, then for $\tilde{P}$ if $u^{(1)}(i) \geqq 0$ for $z_{j} \geqq 1(i, j=1,2,3), \tilde{P} \in \mathscr{\mathscr { L }}^{+}$.

Proof. (i) It is sufficient to consider $P$ given by (4.1). Thus following Lemma 4 and (4.3), we must prove

$$
\begin{array}{ll}
s_{0}+s_{i}+s_{j}-s_{k} \geqq 0, & (i, j, k)=(1,2,3) \\
s_{0}+s_{i} \geqq 0, & (i=1,2,3) \\
s_{0} \geqq 0 &
\end{array}
$$

provided $\left(\beta_{1}, \beta_{2}, \beta_{3}\right) \in \mathscr{L}^{+}$. It is a straightforward calculation. 
(ii) We present an example. For $P$ given by (4.1),

$$
u^{(2)}(1,2)=z_{1} z_{2}\left[\left(\beta_{3}-\beta_{1} \beta_{2}\right)\left(1+z_{3}^{2}\right)+\left(1-\beta_{1}^{2}-\beta_{2}^{2}+\beta_{3}^{2}\right) z_{3}\right] / P^{2} .
$$

Thus obviously $u^{(2)} \geqq 0$ provided $P \in \mathscr{L}^{+}$and $z_{3}=1$. However consider the point $(1 / 3,1 / 3,0) \in \overrightarrow{\mathscr{L}}^{+}$. At this point, $\beta_{3}-\beta_{1} \beta_{2}=-1 / 9$. Hence $u^{(2)}<0$ if $z_{3}$ is large enough.

(iii) By Lemma 4, the necessary and sufficient condition so that $u^{(1)}(i) \geqq 0$ for $z_{j} \geqq 1(i, j=1,2,3)$ is

$$
1+\beta_{i} \geqq \beta_{j}+\beta_{k} \quad(i, j, k)=(1,2,3) .
$$

Finally we investigate the fourth Ursell function $[7,8]$ :

$$
u^{(4)}(1,2,3,4)=\prod_{i=1}^{4}\left(z_{i} \partial / \partial z_{i}\right) \log P .
$$

As is well known, even if $P \in \mathscr{D}^{+}, u^{(4)}$ is not necessarily negative when $z_{i} \geqq 1$, but is negative when $z_{i}=1(i \in \Lambda)$ (the so-called Lebowitz inequality). Contrary to the case of $u^{(2)}, u^{(4)}$ is not necessarily negative even if $P \in \mathscr{L}^{+}$and $z_{i}=1(i \in \Lambda)$. In fact let

Thus

$$
\begin{aligned}
P\left(z_{1}, z_{2}, z_{3}, z_{4}\right)= & P_{\mid z_{5}=\ldots=z_{n}=1} \\
= & \operatorname{const}\left[\left(1+z_{1} \ldots z_{4}\right)+\sum \beta_{i}\left(z_{i}+\partial / \partial z_{i}\left(z_{1} \ldots z_{4}\right)\right)\right. \\
& \left.+\sum \beta_{i j}\left(z_{i} z_{j}+\partial^{2} / \partial z_{i} \partial z_{j}\left(z_{1} \ldots z_{4}\right)\right)\right] .
\end{aligned}
$$

$$
\begin{aligned}
u^{(4)}(1,2,3,4)= & z_{1} z_{2} z_{3} z_{4}\left[P^{3} P_{1,2,3,4}-P^{2} \sum P_{i j} P_{k l}\right. \\
& \left.-P^{2} \sum P_{i} P_{j k l}+2 P \sum P_{i j} P_{k} P_{l}-6 P_{1} P_{2} P_{3} P_{4}\right] / P^{4}
\end{aligned}
$$

and

$$
\begin{aligned}
u^{(4)}(1,2,3,4)_{\mid z=1}= & C\left[\left\{\left(\sum \beta_{i}\right)^{2} / 2-2 \sum \beta_{i} \beta_{j}-1 / 2\right\}\right. \\
& \left.+\left\{\left(\sum \beta_{i j}\right)^{2} / 2-\sum \beta_{i j}^{2}+\sum \beta_{i j}\right\}\right] \text { with } C>0 .
\end{aligned}
$$

The point $\left(\beta_{i}=0, \beta_{i j}=1 / 3\right) \in R^{7}$ is $\in \overline{\mathscr{L}}^{+}$, but at this point [ $]=2 / 3>0$. Thus we see that $P \in \mathscr{L}^{+}$implies neither $u^{(4)} \leqq 0$ with zero external fields nor $u^{(3)} \leqq 0$ with positive external fields (see the next section).

\section{Some Remarks on the Correlation Inequalities}

We have seen that the partition functions which belong to $\mathscr{L}^{+}$do not necessarily satisfy the correlation inequalities expected from the results seen in $P \in \mathscr{D}^{+}$. The reason is obvious, in fact $P \in \mathscr{L}^{+}$is a property which is derived from the behavior of $P$ on $D \otimes D \otimes \ldots \otimes D C C^{n}$, and on the other hand correlation inequalities crucially depend on the behavior of $P$ on $[1, \infty)^{n} C R^{n}$. Our examples suggest

$$
\begin{gathered}
C_{i} \\
\cup \\
\mathscr{D}^{+} \subset \cap_{i} C_{i} \subset \overline{\mathscr{L}}^{+} .
\end{gathered}
$$

On the other hand, Newman showed [5]

$$
(-1)^{l-1}(z \partial / \partial z)^{2 l} \log P(z, z, \ldots, z)_{\mid z=1} \geqq 0 \text { provided } P \in \mathscr{I} \text {. }
$$

This is not unexpected since these are linear combinations of the Ursell functions. 
Our analysis implies that $\mathscr{L}^{+}$-class is too wide to satisfy all the correlation inequalities. Finally we show that the even correlation inequalities with zero external fields follows from the odd correlation inequalities with positive external fields (see also the note-added in [9]).

Theorem 3. For $P \in \mathscr{P}_{e}$, if $u^{(3)}(i, j, k) \leqq 0$ with positive external fields holds, $u^{(4)}(i, j, k, l)_{z=1} \leqq 0$.

Proof. $u^{(4)}(i, j, k, l)=z_{l} \partial / \partial z_{l} u^{(3)}(i, j, k)_{\mid z=1}$.

Since we can put all $z$ except $z_{l}$ equal to 1 in $u^{(3)}$ and $P \in \mathscr{P}_{e}$, then we have

$$
u^{(3)}(i, j, k)=\left(1-z_{l}\right) f\left(z_{l}\right)
$$

where the GHS inequality ensures $f\left(z_{l}\right) \geqq 0$ for $z_{l} \geqq 1$. This completes the proof.

Remark. As is well known, the higher order Ursell functions do not satisfy the expected inequality for $z_{i} \geqq 1$ even for $P \in \mathscr{D}^{+}$. However, if they satisfy the conjectured inequalities (including odd Ursell functions) for $1 \leqq z_{i}<1+\varepsilon$ with $\varepsilon>0$, $i \in \Lambda$, we see that $(-1)^{l-1} u_{z=1}^{(2)} \geqq 0$ can be derived from $(-1)^{l-1} u^{(2 l-1)} \geqq 0$ with $1 \leqq z_{i}<1+\varepsilon$. If this is true, $u^{(2 l)}$ and $u^{(2 l-1)}$ should be considered as a pair. See also the discussions in Lemma 2, and by the same discussions, we see that the converse is true.

Corollary 1. For $P \in \mathscr{L}^{+}, u^{(4)}(i, j, k, l)_{\mid z=1} \leqq 0$ provided that at least two of $(i, j, k, l)$ are equal.

Proof. Case (i) Two arguments are equal: The inequality follows from Theorems 2 and 3 for the case.

Case (ii) Three arguments are equal: Without loss of generality, let $(i, j, k, l)=$ $(1,2,2,2)$. Thus $u_{\mid z=1}^{(4)}=\left(P P_{1,2}-P_{1} P_{2}\right)\left(P^{2}+6 P_{2}^{2}-6 P P_{2}\right) / P^{4}{ }_{\mid z=1}$. This is negative since $P \in \mathscr{L}^{+}$.

Case (iii) All the arguments are equal: The problem reduces to $P=$ const $(1+z)$.

\section{Structure of $\mathscr{L},\{\mathrm{G}-1\}$ and $\mathscr{J}$}

Before studying the topological structure of $\mathscr{L}$, we would like to point out that a product can be defined on $\mathscr{L}[6,10,11]$. We call this product the Asano product.

Theorem 4. Let $\left\{\alpha_{i_{1}, \ldots, i_{l}}^{(l)}\right\} \in \mathscr{L}^{(n)},\left\{\beta_{i_{1}, \ldots, i_{l}}^{(l)}\right\} \in \mathscr{L}^{(n)}$ then $\left\{\alpha_{i_{1}, \ldots, i_{l}}^{(l)} \beta_{i_{1}, \ldots, i_{l}}^{(l)}\right\} \in \mathscr{L}^{(n)}$.

This is a very well known theorem, and we do not repeat the proof. Details are shown in $[6,10,11]$. Therefore $\mathscr{L}$ has a semi-group structure by this product. Remark that $\mathscr{D}$ is also closed under the product. We denote this product by $\{\alpha \beta\}$ or $A\left[P_{\alpha} P_{\beta}\right]$.

The Lee-Yang class $\mathscr{L}$ is a complicated set in the space of the $d$-coefficients $\left(d=2^{n-1}-1\right)$. Let $P$ be given by $(2.1)$, then we identify $P$ with $\left\{\beta_{1}^{(1)}, \ldots, \beta_{1,2}^{(2)}, \ldots\right\} \in R^{d}$ as before. We can easily prove that $\mathscr{L}$ is a bounded set in $R^{d}$. The proof is left to the reader as an exercise. The following theorem is an extention of a Ruelle's result on the openness of $\mathscr{L}$ [6].

Theorem 5. (i) $\mathscr{L}$ is open, arcwise connected.

(ii) $\mathscr{L}$ is homeomorphic to d-dimensional open disk $D^{(d)}$. 
Proof. (i) The openness of $\mathscr{L}$ follows from the definition (see, for example, Ref. [6]). Let $P_{t} \in \mathscr{D}$ be given by putting independent of $i, j \gamma_{i j}=t \in[0,1]$. Then $P_{1} \in \overline{\mathscr{L}}$ and $P_{t} \in \mathscr{L}$ for $t \in[0,1) . P_{t}$ is a continuous line connecting $\prod\left(1+z_{i}\right) \in \overline{\mathscr{L}}$ and $1+\prod z_{i} \in \mathscr{L}$, and lies in $\mathscr{L}$. For any $P \in \mathscr{L}, \tilde{P}_{t}=A\left[P_{t} P\right] \in \mathscr{L}$ is continuous with respect to $t \in[0,1]$, and $\tilde{P}_{1}=P \in \mathscr{L}, \tilde{P}_{0}=1+\prod z_{i} \in \mathscr{L}$. Thus $\mathscr{L}$ is arcwise connected.

(ii) From the above discussions, we see, by operating $A\left[P_{t} \ldots\right]$, that any subset of $\mathscr{L}$ can be continuously contracted to the origin $1+\prod z_{i}$. In other words, $\mathscr{L}$ is contractible. Note that $\mathscr{L}$ is open, bounded. Furthermore, since the mapping $A\left[P_{t} \ldots\right]$ is given by

$$
A\left[P_{t} \ldots\right]:\left\{\beta_{i_{1} i_{2} \ldots i_{l}}^{(l)}\right\} \rightarrow\left\{t^{n} C_{l} \beta_{i_{1} i_{2} \ldots i_{l}}^{(l)}\right\},
$$

we see that $\mathscr{L}$ is homeomorphic to an open, bounded star-like set. Therefore it is sufficient to show that an open, bounded star-like set $U \subset R^{d}$ is homeomorphic to an open disk $D^{(d)}$. Denote by $\bar{U}$ the closure of $U$ with boundary $\partial \bar{U}=\bar{U}-U$, and let $B_{r}$ be an open ball of radius $r$ such that

$$
U \supset \bar{B}_{r} \supset B_{r} \ni\{0\} \text {. }
$$

For any $x \in \partial \bar{B}_{r}$, there is a unique point $\lambda(x) \in \partial \bar{U}$ such that the open line segment $(0, \lambda(x))$ is in $U$. Let $V_{x}$ be an open star-like neighborhood of $(0, \lambda(x))$ in $U$ which itself is a cell. Note that $\partial \bar{U}$ is compact. Let $N_{\varepsilon_{n}}(\partial \bar{U})$ be an open $\varepsilon_{n}$-bounded neighborhood of $\partial \bar{U}$ in $\bar{U}$, and $\lim \varepsilon_{n}=0$. Thus $\partial \bar{U}=\cap_{n} N_{\varepsilon_{n}}(\partial \bar{U})$. Since $\partial \bar{U}$ is compact, we can choose the following type of finite covering of $\bar{U}$ for each $n$ :

$$
\bar{U}=B_{r} \cup N_{\varepsilon_{n}}(\partial \bar{U}) \cup\left\{V_{x_{(n, 1)}} \cup V_{x_{(n, 2)}} \ldots V_{x_{\left(n, k_{n}\right)}}\right\} .
$$

Let $n \rightarrow \infty$, then we have a countable covering of $\bar{U}$ :

$$
\bar{U}=B_{r} \cup \partial \bar{U} \cup\left\{V_{x_{(1,1)}} \cup \ldots V_{x_{\left(1, k_{1}\right)}} \cup V_{x_{(2,1)}} \ldots\right\} .
$$

Therefore $U$ is a countable union of open star-like cells, and we can conclude that $U$ itself is homeomorphic to an open disk. This completes the proof.

Remarks. (i) If the coefficients are complex, these statements can be extended by suitable redefinitions [6].

(ii) Even if $U$ is open, bounded and contractible, $U$ may not be necessarily homeomorphic to an open disk.

The main theorem in this section is:

Theorem 6. (i) $\mathscr{D} \subset \overline{\mathscr{L}} \subset \hat{\mathscr{D}}$.

(ii) $\hat{\mathscr{D}}=\hat{\mathscr{L}}$.

Proof. (i) $\mathscr{D} \subset \overline{\mathscr{L}}$ is well known. Consider the following $(d+1)$ functions:

$$
\prod^{n}\left(1 \pm z_{i}\right)
$$

where the number of $(-)$ signs is even, and which ensures that these functions belong to $\mathscr{P}_{e}$. We denote these functions by $P_{i}(i=1, \ldots, d+1)$, and remark that $P_{i} \in \mathscr{D}\left(P_{i} \in \overline{\mathscr{L}}\right)$ and $d$-tuple of $P$ 's are linearly independent. Then $P=\sum \alpha_{i} P_{i}$ with $\alpha_{i} \geqq 0, \sum \alpha_{i}=1$ becomes a $d$-dimensional convex cell in the $d$-dimensional space of the coefficients. Thus, denoting this convex cell by $\hat{\mathscr{D}}^{\prime}$, we show $\partial \hat{\mathscr{D}}^{\prime} \cap \mathscr{L}=\phi$. 
Once this is proved, (i) follows from Theorem 5 and the fact $1+\prod z_{i}=(d+1)^{-1} \sum P_{i}$ $\in \hat{\mathscr{D}} \cap \mathscr{L}$. Each hypersurface of $\partial \hat{\mathscr{D}}^{\prime}$ is a $(d-1)$-dimensional convex cell. We rewrite $P$ as

$$
P=P_{\mid z_{n}=0}+z_{n} \partial / \partial z_{n} P=B\left(z_{1}, \ldots, z_{n-1}\right)+z_{n} A\left(z_{1}, \ldots, z_{n-1}\right) .
$$

Since $P \in \mathscr{L}$ is equivalent to $|A / B|<1$ provided $z_{i} \in D$ and some $z_{j} \in D^{0}$, it is necessarily $B \neq 0$ when $\left(z_{1}, \ldots, z_{n-1}\right) \in \otimes^{n-1} D . B$ is given by $\sum \alpha_{i} P_{i \mid z_{n}=0}$, and consider the point $\left(z_{1}, \ldots, z_{n-1}\right)=( \pm 1, \pm 1, \ldots, \pm 1)$. There are $2^{n-1}=d+1$ points. For the given point the function which does not vanish at the point is one of the following two possible functions:

$$
\begin{aligned}
& \prod_{i=1}^{i=n-1}\left(1 \pm z_{i}\right)\left(1+z_{n}\right), \\
& \prod_{i=1}^{i=n-1}\left(1 \pm z_{i}\right)\left(1-z_{n}\right),
\end{aligned}
$$

and only one of these functions belong to $\mathscr{P}_{e}$. Thus any $d$-tuple of $P$ 's vanish simultaneously at one point of these points. Therefore $\partial \hat{\mathscr{D}}^{\prime} \cap \mathscr{L}=\phi$, and (i) follows.

(ii) This is obvious from the above discussions.

Corollary 2. (i) Let $\{G-1\}$ be a set of $P$ in $\mathscr{P}_{e}$ which obeys the Griffiths first inequality including the cases such that the relevant correlation functions become $+\infty$. Then

$$
\mathscr{D} \subset \overline{\mathscr{L}} \subset \hat{\mathscr{D}}=\hat{\mathscr{L}} \subset\{G-1\} \text {. }
$$
Then

(ii) Let $\{G-1\}^{+}$be a set of $P$ in $\mathscr{P}_{e}^{+}$which obeys the Griffiths first inequality.

$$
\hat{\mathscr{D}}^{+} \subset \hat{\mathscr{L}}^{+} \subset\{G-1\}^{+} \subset C_{1} .
$$

Proof. (i) Let $P_{i} \in \mathscr{L}$ and note that $P_{i}(0, \ldots, 0)=1$ by definition. Then $P_{i}>0$ for $z_{k} \geqq 1(k \in \Lambda)$. Furthermore, by Theorem 1 , for any subset $S \subset \Lambda$

$$
\left(\prod_{j \in S} \partial / \partial z_{j}\right)\left[P_{i} \prod_{k \in \Lambda} z_{k}^{-1 / 2}\right]=Q_{i}\left[2^{|S|} \prod_{k \in \Lambda} z_{k}^{1 / 2}\right]^{-1} \geqq 0
$$

for $z_{k} \geqq 1(k \in \Lambda)$. Therefore for $P=\sum \alpha_{i} P_{i} \in(\hat{\mathscr{L}})^{0}$ with $\sum \alpha_{i}=1, \alpha_{i} \geqq 0$, Thus

$$
u \equiv\left\{\left(\prod_{j \in S} z_{j} \partial / \partial z_{j}\right)\left[P \prod_{k \in \Lambda} z_{k}^{-1 / 2}\right]\right\}\left[P \prod_{k \in \Lambda} z_{k}^{-1 / 2}\right]^{-1}=\left(\sum \alpha_{i} Q_{i}\right) /\left(2^{|S|} P\right) .
$$

$\infty>u \geqq 0$ provided $z_{k} \geqq 1(k \in \Lambda)$ and $P_{i} \in \mathscr{L}(i=1,2, \ldots)$.

(ii) This is obvious from the above discussions.

Conjecture. $\mathscr{D}^{+} \subset \overline{\mathscr{L}}^{+} \subset \hat{\mathscr{D}}^{+}, \hat{\mathscr{D}}^{+}=\hat{\mathscr{L}}^{+}$.

Remark. One may define the vertices of $\mathscr{D}, \mathscr{L}$. In order to define the vertices, however, we must use the terminologies of algebraic geometry. Since $\mathscr{D}$ and $\mathscr{L}$ are semi-analytic sets, we can define the vertices as the zero-dimensional singularities of $\partial \mathscr{D}, \partial \overline{\mathscr{L}}$. This is usually done through the stratification of the singularity. We conjecture

(i) $\operatorname{Ver} \mathscr{D}=\operatorname{Ver} \overline{\mathscr{L}}$,

(ii) $\operatorname{Ver} \mathscr{D}^{+}=\operatorname{Ver} \overline{\mathscr{L}}^{+}$.

These can be confirmed easily for $n=1,2,3$. 
Finally we comment on some interesting properties of $\mathscr{I}$. Let $P(z, \ldots, z)=$ $\left(1+z^{n}\right)+a_{1}\left(z+z^{n-1}\right)+a_{2}\left(z^{2}+z^{n-2}\right)+\ldots \in \mathscr{I}$. Recently Millard et al. have obtained a generalization of the Ruelle's lemma $[6,12]$ :

Theorem 7. Let $A$ and $B$ be closed circular regions (see [12]) not containing the origin. If $f=\sum_{i=0}^{n} b_{i} z^{i}$ vanishes only in $A \subset C$, and $g=\sum_{i=0}^{n} c_{i} z^{i}$ vanishes only in $B \subset C$, then $A[f g] \equiv \sum_{i=0}^{n}{ }_{n} C_{i}^{-1} b_{i} c_{i} z^{i}$ vanishes only in $A B=\left\{z \in C ; z=-z_{1} z_{2}, z_{1} \in A\right.$, $\left.z_{2} \in B\right\}$.

Therefore, using the same techniques in Theorem 5, we have:

Theorem 8. (i) $\mathscr{I}$ is a closed, contractible set.

(ii) Let $\mathscr{F}_{S}=\left\{z^{n}+a_{n-1} z^{n-1}+\ldots+a_{1} z+1 ;\left(a_{n-1}, \ldots, a_{1}\right) \in R^{n-1}\right\}$ be functions whose roots are all in an open region $S \subset C$ which is invariant under the rotation around the origin. Then $\mathscr{F}_{S}$ is homeomorphic to $(n-1)$-dimensional open disk $D^{(n-1)}$.

Acknowledgements. The author is deeply indebted to Prof. H. Araki for the encouragement. He also thanks Prof. M. Suzuki for sending to the author his beautiful papers. Discussions with Prof. J. Glimm at the International Symposium on Mathematical Problems in Theoretical Physics (Kyoto, 1975) were very fruitful. The proof of Theorem 5 (ii) is essentially due to Prof. Shimada. The author's colleagues, Drs. N. Seto and A. Kishimoto should be acknowledged for the fruitful discussions.

\section{References}

1. Guerra,F., Rosen, L., Simon, B.: Ann. Math. 101, 111 (1975);

Simon, B.: The $P(\phi)_{2}$ Euclidean (Quantum) Field Theory. Princeton: University Press 1974, and the further references cited therein

2. Simon, B., Griffiths, R.B.: Commun. math. Phys. 33, 145 (1973)

3. Ruelle, D.: Statistical Mechanics, Rigorous Results. New York: Benjamin Inc. 1969. The old references before 1969 are cited therein

4. Griffiths, R. B., Hurst, C. A., Sherman, S.: J. Math. Phys. 11, 790 (1970)

5. Newman, C.M.: Commun. math. Phys. 41, 1 (1975); Indiana Preprint. Gaussian Correlation Inequalities for Ferromagnets

6. Ruelle, D.: Phys. Rev. Letters 26, 303 (1971); Commun. math. Phys. 31, 265 (1973)

7. Lebowitz, J.L.: Commun. math. Phys. 35, 87 (1974)

8. Sylvester, C.S.: MIT-preprint

9. Ellis, R. S., Monroe, J. L.: Commun. math. Phys. 41, 33 (1975)

10. Asano, T.: Phys. Rev. Letters 24, 1409 (1970)

11. Suzuki, M., Fisher, M. E.: J. Math. Phys. 21, 235 (1971)

12. Millard, K.Y., Viswanathan, K. S.: J. Math. Phys. 15, 1821 (1974)

Communicated by G. Gallavotti 
